\title{
Wigner Method in Quantum Statistical Mechanies*
}

\author{
KaYa IMRE† AND ERcüMENT OZIZMIR: \\ Cekmece Nuclear Research and Training Center, Istanbul, Turkey \\ Marcos Rosenbaum \\ Department of Nuclear Engineering, The University of Michigan, Ann Arbor, Michigan \\ P. F. ZWEIFEL \\ Department of Theoretical Physics, Middle East Technical University, Ankara, Turkey, \\ and Department of Nuclear Engineering, The University of Michigan, Ann Arbor, Michigan
}

(Received 28 March 1966)

\begin{abstract}
The Wigner method of transforming quantum-mechanical operators into their phase-space analogs is reviewed with applications to scattering theory, as well as to descriptions of the equilibrium and dynamical states of many-particle systems. Inclusion of exchange effects is discussed.
\end{abstract}

\section{INTRODUCTION}

$\mathrm{T}$ HE ensemble expectation value of a quantummechanical operator $A$ is expressed in the usual formulation of quantum statistics, as the trace of $\rho A$, namely,

$$
\langle A\rangle=\operatorname{Tr} \rho A,
$$

where $\rho$ is the von Neumann density matrix ${ }^{1}$ defined by

$$
\rho=\sum_{i} w_{i}\left|\phi_{i}(t)\right\rangle\left\langle\phi_{i}(t)\right|,
$$

where $w_{i}$ is the probability that the system will be in the state $\left|\phi_{i}(t)\right\rangle$. The density matrix satisfies the equation

$$
i \hbar(\partial \rho / \partial t)=H \rho-\rho H,
$$

where $H$ is the Hamiltonian of the system under consideration. In equilibrium, for a canonical ensemble one has

$$
\rho=e^{-\beta H} / \operatorname{Tr} e^{-\beta H} .
$$

In calculating expectation values of physical interest one may choose any convenient representation in which to work. Wigner ${ }^{2}$ in 1932 introduced a method for evaluating expectation values which is particularly suitable for "almost classical" systems in that it expresses the expectation values as a power

* The portion of this work carried out at the University of Michigan was supported by the U.S. Atomic Energy Commission. Some of this material is based on portions of a thesis of one of the authors (M. R.) presented to the University of Michigan in partial fulfillment of the requirements for the Ph.D. degree.

† Present address: Conductron Corporation, Ann Arbor, Michigan.

$\ddagger$ Present address: Plasma Physics Laboratory, Princeton, New Jersey.

8 Present address: General Electric Company, TEMPO, Santa Barbara, California.

II On sabbatical leave, academic year 1964-1965 at Middle East Technical University. Permanent address: Department of Nuclear Engineering, The University of Michigan, Ann Arbor, Michigan.

1 J. von Neumann, Mathematical Foundation of Quantum Mectianics (Princeton University Press, Princeton, New Jersey, 1955).

E. Wigner, Phys. Rev. 40, 749 (1932). series expansion with respect to Planck's constant. For such systems the expansion may be expected to converge rapidly. Another important advantage of this method is that there are direct classical analogs of the quantities and operations used. In particular, the analog of classical phase space is introduced into quantum statistics. In this way the expectation values of physical variables may be expressed in terms of a phase-space integration. The purpose of the present study is to review various fields of application of this method. ${ }^{3}$

In Sec. II, we demonstrate that the Wigner method can be defined as a means for associating a $c$-number function in phase space with every operator which is a function of position and momentum operators. This rule, in fact, is the inverse of Weyl's rule, which is used to calculate quantum-mechanical operators from classical quantities. However, it is interesting to observe that there are various equivalent ways of stating the association which are, in many cases, simpler than Weyl's rule. Various properties and applications of this correspondence can be found in Sec. III. Section IV is devoted to the application to scattering theory. A method of inclusion of exchange effects in the previous results is given in Sec. $V$, where the second quantized formalism is also discussed.

Application to the equilibrium case (for Boltzmann statistics) is given in Appendixes A and B, where the equation of state is derived up to the order $\hbar^{4}$.

There are several papers published which deal with the Wigner distribution function. Some of the basic references are: $H$. J. Groenewold, Physica 12, 405 (1946); J. E. Moyal, Proc. Cambridge Phil. Soc. 45, 99 (1949); J. H. Irving and R. W. Zwanzig, J. Chem. Phys. 19, 1173 (1951); H. Mori, I. Oppenheim, and J. Ross, in Studies in Statistical Mechanics, J. de Boer and G. E. Uhlenbeck, Eds. (North-Holland Publishing Company, Amsterdam, 1962), Vol. 1. 


\section{WIGNER DISTRIBUTION FUNCTION}

We restrict ourselves to Boltzmann statistics in this section, so that exchange effects are ignored. Also, we assume that the Hamiltonian of our system depends only on the position and momentum operators $R$ and $P .4$

Wigner ${ }^{2}$ defines a distribution function $f_{w}(r, p)$ as the Fourier transform of the off-diagonal elements of the density matrix

$$
\begin{aligned}
f_{w}(r, p)=(2 \pi \hbar)^{-3 N} \int d z & \exp (i p \cdot z / \hbar) \\
\times & \left\langle r-\frac{z}{2}|\rho| r+\frac{z}{2}\right\rangle \\
=(2 \pi \hbar)^{-3 N} \int d k & \exp (-i r \cdot k / \hbar) \\
\times & \left\langle p-\frac{k}{2}|\rho| p+\frac{k}{2}\right\rangle .
\end{aligned}
$$

It is easily verified that $f_{w}$ has the following properties :

and, evidently

$$
\begin{aligned}
& \int d p f_{w}(r, p)=\langle r|\rho| r\rangle, \\
& \int d r f_{w}(r, p)=\langle p|\rho| p\rangle,
\end{aligned}
$$

$$
\int d r d p f_{w}(r, p)=\operatorname{Tr} \rho=1 .
$$

Corresponding to a quantum operator $A(R, P)$, we define a function $A_{w}(r, p)$ by an equation analogous to (5):

$$
\begin{aligned}
A_{w}(r, p) & =\int d z \exp (i p \cdot z / \hbar)\left\langle r-\frac{z}{2}|A| r+\frac{z}{2}\right\rangle \\
& =\int d k \exp (-i r \cdot k / \hbar)\left\langle p-\frac{k}{2}|A| p+\frac{k}{2}\right\rangle,
\end{aligned}
$$

which we call the Wigner equivalent of $A$. Thus we see that $f_{w}$ is simply $(2 \pi \hbar)^{-3 N}$ times the Wigner equivalent of the density matrix $\rho$ :

$$
f_{w}=(2 \pi \hbar)^{-3 N} \rho_{w} .
$$

Furthermore, from (5) and (7)

$$
\operatorname{Tr} \rho A=\int d r d p A_{w}(r, p) f_{w}(r, p)
$$

\footnotetext{
In our notation, $r, p$ represent $3 N$-dimensional vector $c$ numbers for position and momentum variables and $R, P$ represent the corresponding vector operators. A $3 \mathrm{~N}$-dimensional scalar product is written as $\boldsymbol{R} . \boldsymbol{P}$ or r.p. Also, $\mathbf{R}_{i}, \mathbf{P}_{i}: \mathbf{x}_{i}, \mathbf{p}_{i}$, etc. denote ordinary threo-dimensional vectors associated with the $i$ th particle.
}

Equation (9) is the key result of the Wigner method, since it expresses the ensemble average of an operator $A$ as a phase space integral.

The rules (5) or (7) for Wigner equivalent operators are actually equivalent to Weyl's rule ${ }^{5}$ for defining the classical analog of a quantum operator. This rule may be stated most conveniently starting with the Fourier transform of the classical function $A_{w}(r, p)$ :

$$
\alpha(\sigma, \tau)=\int d r d p \exp [-i(\sigma \cdot r+\tau \cdot p) / \hbar] A_{w}(r, p) .
$$

Then $A(R, P)$ is defined from

$$
\begin{aligned}
A(R, P)= & \left(\frac{1}{2 \pi \hbar}\right)^{6 N} \\
& \times \int d \sigma d \tau \exp [i(\sigma \cdot R+\tau \cdot P) / \hbar] \alpha(\sigma, \tau) .
\end{aligned}
$$

That the Weyl rules (10) relating $A$ and $A_{w}$ are identical with the Wigner rules, (7) is shown below. [Also, we show that Eqs. (10) "work both ways". Given $A_{w}$ one may determine $A$, and vice versa.] There has apparently been some confusion in the literature, in which one frequently finds the statement that Eq. (9) holds with $f_{w}$ defined through Eq. (5) and $A_{w}$ through (10).

The equivalence of (7) and (10) may be proved as follows. We begin by proving the completeness (and orthogonality) of the operators $\exp [i(\sigma \cdot R+\tau \cdot P)] / \hbar$ in the space of operators of the form $A=A(R, P)$. We first write

$$
\begin{aligned}
& \exp [i(\sigma \cdot R+\tau \cdot P) / \hbar] \\
& \quad=\exp [i \sigma \cdot R / \hbar] \exp [i \tau \cdot P / \hbar] \exp [i \sigma \cdot \tau / 2 \hbar],
\end{aligned}
$$

by making use of the identity $e^{A+B}=e^{A} e^{B} e^{\frac{1}{2}[B, A]}$ (true if the commutator $[B, A]$ commutes with both $A$ and $B)$. Since $\exp (i \tau \cdot p \mid \hbar)|r\rangle=|r-\tau\rangle$, we have

$$
\begin{aligned}
& \left\langle r^{\prime}|\exp [ \pm i(\sigma \cdot R+\tau \cdot P) / \hbar]| r\right\rangle \\
& \quad=\exp \left[ \pm i \sigma \cdot\left(r \mp \frac{1}{2}\right) / \hbar\right] \delta\left(r^{\prime}-r \pm \tau\right),
\end{aligned}
$$

which implies that

$\operatorname{Tr} \exp [-i(\sigma \cdot R+\tau \cdot P) / \hbar]=(2 \pi \hbar)^{3 N} \delta(\sigma) \delta(\tau)$.

Therefore

$$
\begin{gathered}
\operatorname{Tr} \exp \left[-i\left(\sigma^{\prime} \cdot R+\tau^{\prime} \cdot P\right) / \hbar\right] \exp [i(\sigma \cdot R+\tau \cdot P) / \hbar] \\
=\operatorname{Tr} \exp \left\{-i\left[\left(\sigma^{\prime}-\sigma\right) R+\left(\tau^{\prime}-\tau\right) P\right] / \hbar\right\} \\
\times \exp \left[-\frac{1}{2} \frac{i}{\hbar}\left(\tau^{\prime} \cdot \sigma-\sigma^{\prime} \cdot \tau\right)\right], \quad(14 \mathrm{a}) \\
=(2 \pi \hbar)^{3 N} \delta\left(\sigma^{\prime}-\sigma\right) \delta\left(\tau^{\prime}-\tau\right)
\end{gathered}
$$

H. Weyl, The Theory of Groups and Quantum Mechanics (Dover Publications, New York, 1950). 
[Equation (14a) was obtained trivially using the identity below Eq. (11).]

We thus have proved that the operators

$$
(2 \pi h)^{-3 N / 2} \exp [i(\sigma \cdot R+\tau \cdot p) / \hbar]
$$

are orthonormal. To prove completeness we attempt to expand an arbitrary operator $A(R, P)$ in terms of these functions:

$A(R, P)=\int d \sigma d \tau \alpha(\sigma, \tau) \exp [i(\sigma \cdot R+\tau \cdot P) / \hbar]$.

If the expansion exists then the coefficient $\alpha(\sigma, \tau)$ can be calculated by multiplying Eq. (15) by $\exp \left[-i\left(\sigma \cdot R^{\prime}+\tau \cdot p^{\prime}\right)\right]$ and using Eq. (14):

$$
\begin{aligned}
& a(\sigma, \tau)=(2 \pi \hbar)^{-3 N} \\
& \quad \times \operatorname{Tr}\left\{A\left(R^{\prime}, P^{\prime}\right) \exp \left[-i\left(\sigma \cdot R^{\prime}+\tau \cdot P^{\prime}\right) / \hbar\right]\right\} .
\end{aligned}
$$

To prove that the expansion (15) exists, i.e., to prove completeness, we substitute from (10) into (15) and prove that the result is an identity, say by taking matrix elements in the position representation.

$$
\begin{aligned}
\left\langle r|A| r^{\prime}\right\rangle= & (2 \pi \hbar)^{-3 N} \int d \sigma d \tau d r^{\prime \prime} d r^{\prime \prime \prime}\left\langle r^{\prime \prime}|A| r^{\prime \prime \prime}\right\rangle \\
& \times\left\langle r^{\prime \prime \prime}\left|\exp \left[-i\left(\sigma \cdot R^{\prime}+\tau \cdot P^{\prime}\right) / \hbar\right]\right| r^{\prime \prime}\right\rangle \\
& \times\left\langle r|\exp [i(\sigma \cdot R+\tau \cdot P) / \hbar]| r^{\prime}\right\rangle .
\end{aligned}
$$

Making use of Eq. (13) and carrying out the trivial integration, Eq. (17) is seen to reduce to the identity

$$
\left\langle r|A| r^{\prime}\right\rangle=\left\langle r|A| r^{\prime}\right\rangle,
$$

which proves completeness (in the weak topological sense at least).

This proof permits us to consider Eqs. (10) to work in either direction, i.e., given $A(R, P)$, then $A_{w}(r, p)$ can be found and vice versa. Thus there exists a oneto-one correspondence between $A(R, P)$ and the $c$-number functions $A_{w}(r, p)$.

Finally, it is trivial to prove now that definitions (7) and (10) are equivalent. It is only necessary to substitute the expansion (10b) into (7a), use Eq. (12), and carry out the trivial integration, whereupon the Fourier inverse of $(10 \mathrm{a})$ is obtained.

In the next section we consider various properties of the correspondence between $A(R, P)$ and $A_{w}(r, p)$. In particular, we show a third rule for defining this equivalence (Groenewold's rule) which frequently is simpler to apply than either Wigner's or Weyl's rules [Eqs. (7) and (10), respectively]. In summarizing the results of the present section we have shown that given any two operators $A(R, P)$ and $B(R, P)$ that

$$
\operatorname{Tr} A B=(2 \pi \hbar)^{-s N} \int d r d p A_{v 0}(r, p) B_{v p}(r, p),
$$

where $A_{w}$ and $B_{w}$ are related to $A$ and $B$ through Wigner's rule (7) or equivalently Weyl's rule (10). In particular, the Wigner distribution function, $f_{w}(r, p)$ is simply $(2 \pi \hbar)^{-3 N} \rho_{w}$, where $\rho_{w}$ is the Wigner equivalent of the density matrix.

\section{WIGNER EQUTVALENT OF OPERATORS}

From the results of the previous section, we immediately deduce the following properties:

(a) if $A=A(P)$ (i.e., independent of $R$ ),

(b) if $A=A(R)$, then $A_{w}=A(r)$;

then $A_{v}=A(p)$;

(c) if $A=$ const, then $A_{w}=A$;

(d) $\operatorname{Tr} A=(2 \pi \hbar)^{-3 N} \int d r d p A_{w}(r, p)$;

(e) $\int d p A_{w}(r, p)=(2 \pi \hbar)^{3 N}\langle r|A| r\rangle$;

(f) $\int d r A_{w}(r, p)=(2 \pi \hbar)^{3 N}\langle p|A| p\rangle$;

(g) $\left\langle r|A| r^{\prime}\right\rangle=(2 \pi h)^{-3 N} \int d p \exp \left[i p \cdot\left(r-r^{\prime}\right) / \hbar\right]$

$$
\begin{aligned}
\times A_{w}\left(\frac{1}{2}\left(r+r^{\prime}\right), p\right) & \\
=(2 \pi \hbar)^{-6 N} \int d \sigma \exp & {\left[i \sigma \cdot\left(r+r^{\prime}\right) / 2 \hbar\right] } \\
\times & \alpha\left(\sigma, r^{\prime}-r\right) ;
\end{aligned}
$$

where $\alpha(\sigma, \tau)$ is the Fourier transform of $A_{w}(r, p)$ as in Eq. (10b).

\section{Wigner Equivalent of Products}

Next we consider the Wigner equivalent of a product of operators $A B$, and derive a formula which expresses $(A B)_{w}$ in terms of $A_{w}$ and $B_{w}$. We have

$$
\begin{aligned}
(A B)_{w}= & \int d z \exp (i p \cdot z / \hbar)\left\langle r-\frac{1}{2} z|A B| r+\frac{1}{2} z\right\rangle \\
= & \int d z d r^{\prime} \exp (i p \cdot z \mid \hbar) \\
& \times\left\langle r-\frac{1}{2} z|A| r^{\prime}\right\rangle\left\langle r^{\prime}|B| r+\frac{1}{2} z\right\rangle,
\end{aligned}
$$

or

$$
\begin{aligned}
(A B)_{w}= & (2 \pi \hbar)^{-12 N} \int d z d r^{\prime} \exp (i p \cdot z / \hbar) \\
& \times \int d \sigma d \sigma^{\prime} \exp \left[i \sigma \cdot\left(r+r^{\prime}-\frac{1}{2} z\right) / 2 \hbar\right] \\
& \times \alpha\left(\sigma, r^{\prime}-r+\frac{1}{2} z\right) \\
& \times \exp \left[i \sigma^{\prime} \cdot\left(r^{\prime}+r+\frac{1}{2} z\right) / 2 \hbar\right] \\
& \times \beta\left(\sigma^{\prime}, r-r^{\prime}+\frac{1}{2} z\right) .
\end{aligned}
$$

Here we have used Eq. (20g) for both $A$ and $B$. Now, making the change of variables

$$
\tau=r^{\prime}-r+\frac{1}{2} z, \quad \tau^{\prime}=r-r^{\prime}+\frac{1}{2} z,
$$


we readily obtain

$$
\begin{aligned}
(A B)_{w}= & (2 \pi \hbar)^{-12 N} \int d \sigma d \sigma^{\prime} d \tau d \tau^{\prime} \\
& \times \exp [i(\sigma \cdot r+\tau \cdot p) / \hbar] \alpha(\sigma, \tau) \\
& \times \exp \left[i\left(\sigma^{\prime} \cdot \tau-\sigma \cdot \tau^{\prime}\right) / 2 \hbar\right] \beta\left(\sigma^{\prime}, \tau^{\prime}\right) \\
& \times \exp \left[i\left(\sigma^{\prime} \cdot r+\tau^{\prime} \cdot p\right) / \hbar\right] .
\end{aligned}
$$

The factor $\exp \left[i\left(\sigma^{\prime} \cdot \tau-\sigma^{\cdot} \tau^{\prime}\right) / 2 \hbar\right]$ in the latter integrand can be replaced by $\exp (\hbar \Lambda / 2 i)$, where $\Lambda$ is the Poisson bracket operator, i.e.,

$$
\Lambda=\overleftarrow{\nabla}_{p} \cdot \vec{\nabla}_{r}-\overleftarrow{\nabla}_{r} \cdot \vec{\nabla}_{p},
$$

so that $A_{w} \Lambda B_{w}=\left(B_{w} ; A_{w}\right)$ is the standard classical Poisson bracket. (The arrows on the $3 N$-dimensional gradient operators indicate the direction in which they operate.) We, therefore, obtain the formulas

$$
\begin{aligned}
(A B)_{w} & =A_{w}(r, p) \exp (\hbar \Lambda / 2 i) B_{w}(r, p) \\
& =B_{w}(r, p) \exp (-\hbar \Lambda / 2 i) A_{w}(r, p) \\
& =A_{w}\left(r-(\hbar / 2 i) \nabla_{p}, p+(\hbar / 2 i) \nabla_{r}\right) B_{w}(r, p)
\end{aligned}
$$

The above result is due to Groenewold, ${ }^{6}$ which, by successive application, along with (20a)-(20c) permits one to calculate the Wigner equivalent of any operator. This rule, then is equivalent to the two rules (Wigner's and Weyl's) described in the previous section. In particular, from Eq. (20d)

$$
\begin{aligned}
\operatorname{Tr} A B & =(2 \pi \hbar)^{-3 N} \int d r d p A_{w}(r, p) \exp (\hbar \Lambda / 2 i) B_{w}(r, p) \\
& =(2 \pi \hbar)^{-3 N} \int d r d p A_{w}(r, p) B_{w}(r, p)
\end{aligned}
$$

To obtain the latter, we have performed $6 N$ partial integrations on the former, which flip the arrows pointing to the left to the right, thus making $\Lambda \rightarrow 0$. This result thus agrees with Eq. (19).

We see that $A_{w}$ is expressed as a power series in $\hbar$. Similarly $f_{w}$ is so expressed and, in particular, so are thermal expectation values.

\section{Wigner Equivalent of Heisenberg Operators}

For $A(t)=\exp (i t H / \hbar) A(0) \exp (-i t H / \hbar)$, one has

$$
\partial A(t) / \partial t=(i / \hbar)(H A-A H) .
$$

Thus, upon forming the Wigner equivalent, we obtain

$$
\begin{aligned}
\partial A_{w}(t) / \partial t= & (i / \hbar)\left[H_{w} \exp (\hbar \Lambda / 2 i) A_{w}\right. \\
& \left.\quad-A_{w} \exp (\hbar \Lambda / 2 i) H_{w}\right] \\
= & (i / \hbar)\left[H_{w} \exp (\hbar \Lambda / 2 i) A_{w}\right. \\
& \left.\quad-H_{w} \exp (-\hbar \Lambda / 2 i) A_{w}\right] \quad(28 \mathrm{a})
\end{aligned}
$$

${ }^{6}$ H. J. Groenewold, Ref. 3; several properties of the Wigner method have been first given in this work. or

$$
\partial A_{v 0}(t) / \partial t=(2 / \hbar) H_{w} \sin (\hbar \Lambda / 2) A_{v o}(t)
$$

Integration gives the formula

$$
A_{w o}(t)=\exp \left[(2 t / \hbar) H_{w} \sin (\hbar \Lambda / 2)\right] A_{v o}(0) .
$$

To lowest order in $\hbar$ this is simply the classical equation of motion.

\section{Quantum Liouville and Bloch Equations}

The so-called quantum Liouville equation, which determines the time evolution of the Wigner distribution function, can be deduced readily by forming the Wigner equivalent of Eq. (3) with the aid of Eq. (8). By similar manipulations used to obtain Eq. (28b), we get

$$
\partial f_{w}(t) / \partial t=-(2 / \hbar) H_{w} \sin (h \Lambda / 2) f_{w o}(t),
$$

which can be solved formally as

$$
f_{w}(t)=\exp \left[-(2 t / \hbar) H_{w} \sin (\hbar \Lambda / 2)\right] f_{w}(0) \text {. }
$$

To the lowest order in $\hbar$, the above equations reduce to the classical Liouville equation:

$$
\begin{aligned}
\partial f_{w}^{C} / \partial t & =-H_{w} \Lambda f_{w}^{C} \Rightarrow f_{w}^{C} \\
& =\exp \left(-t H_{w} \Lambda\right) f_{w}^{C}(0) .
\end{aligned}
$$

Equation (30) may be solved in powers of $\hbar^{2}$ starting from the classical distribution function. Such a procedure has, in fact, been followed by Wigner ${ }^{2}$ and by Irving and Zwanzig. ${ }^{3}$

For a canonical ensemble in equilibrium, one has [cf. Eq. (4)],

$$
\rho Z(\beta)=\Omega=e^{-\beta H},
$$

where $\Omega$ is the so-called unnormalized density matrix and $Z(\beta)$ is the partition function. Forming the Wigner equivalent, we get

$$
\Omega_{w}=\left(e^{-\beta H}\right)_{w},
$$

which may be evaluated as a power series expansion with respect to an appropriate parameter. However, there is another way to handle this evaluation which is suitable for almost-classical systems, and which makes use of the equation?

$$
\partial \Omega / \partial \beta=-H \Omega=-\Omega H .
$$

The Wigner equivalent of the latter is

$$
\begin{aligned}
\partial \Omega_{w} / \partial \beta & =-H_{w} \exp (\hbar \Lambda / 2 i) \Omega_{w} \\
& =-\Omega_{w} \exp (\hbar \Lambda / 2 i) H_{w} \\
& =-H_{w} \exp (-\hbar \Lambda / 2 i) \Omega_{w} \\
\partial \Omega_{w} / \partial \beta & =-H_{w} \cos (\hbar \Lambda / 2) \Omega_{w},
\end{aligned}
$$

${ }^{7}$ This approach has been used by I. Oppenheim and J. Ross, Phys. Rev. 107, 28 (1957). 
with

$$
\Omega_{v}(\beta=0)=1 \text {. }
$$

Equation (37a) is known as the Bloch equation, which provides a means for calculating $\Omega_{w}$ (and thus $f_{w}$ ) in powers of $\hbar^{2}$, which calculation may be found in Appendix A. In this appendix, this method is employed to deduce the $\hbar^{4}$ correction in the equation of state.

\section{APPLICATION TO SCATTERING THEORY}

The differential scattering cross section (in first Born approximation) for a system of interacting particles can be written in the form 8

$$
\partial^{2} \sigma / \partial \Omega \partial \epsilon=\operatorname{CS}(\mathbf{q}, \epsilon),
$$

where $C$ is a factor depending upon the momenta of the incoming and outgoing particles and upon the scattering potential for neutron scattering (which for neutron scattering may be taken as the Fermi pseudopotential ${ }^{\vartheta}$ ). Also, $\epsilon=\hbar w$ and $q=\hbar x$ are the energy and momentum changes in the scattering event. It is customary to express $S(\mathbf{q}, \epsilon)$ (called the "scattering law") in terms of Fourier transformed functions $\chi(\mathbf{q}, t)$ and/or $G(\mathbf{r}, t)$ as

$$
\begin{aligned}
S(\mathbf{q}, \epsilon)= & (2 \pi \hbar)^{-1} N \int d t \exp (-i \epsilon t / \hbar) \chi(\mathbf{q}, t) \\
= & (2 \pi \hbar)^{-1} N \int d^{3} r d t \\
& \quad \times \exp [i(\mathbf{q} \cdot \mathbf{r}-\epsilon t) \hbar] G(\mathbf{r}, t) .
\end{aligned}
$$

Thus $\chi$ and $G$ are related by

or

$$
\chi(\mathbf{q}, t)=\int d^{3} r \exp (i \mathbf{q} \cdot \mathbf{r}) G(\mathbf{r}, t)
$$

$$
G(\mathbf{r}, t)=(2 \pi \hbar)^{-3} \int d^{3} q \exp (-i q \cdot \mathbf{r}) \chi(\mathbf{q}, t)
$$

Explicitly $\chi$ and $G$ are related to the density fluctuations of the scattering system:

$$
\begin{array}{r}
G(\mathbf{r}, t)=\left\langle\frac{1}{N} \sum_{i, j=1}^{N} \int d^{3} r^{\prime} \delta\left(\mathbf{r}+\mathbf{R}_{i}(0)-\mathbf{r}^{\prime}\right)\right. \\
\left.\times \delta\left(\mathbf{r}^{\prime}-\mathbf{R}_{j}(t)\right)\right\rangle, \\
\chi(\mathbf{q}, t)=\left\langle\frac{1}{N} \sum_{i j} \exp \left[-i q \cdot \mathbf{R}_{i}(0) / \hbar\right]\right. \\
\left.\times \exp \left[i \mathbf{q} \cdot \mathbf{R}_{j}(t) / \hbar\right]\right\rangle,
\end{array}
$$

\footnotetext{
${ }^{8}$ L. Van Hove, Phys. Rev. 95, 249 (1954).

E. Fermi, Ric. Sci. 7, 13 (1938); G. C. Summerfield, Ann. Phys. (N.Y.) 26, 72 (1964).
}

where $\mathbf{R}_{j}(t)$ is the Heisenberg position operator corresponding to the $j$ th scattering center (out of the total $N$ ).

For almost classical systems, it is desirable to relate the cross section to the classical time-dependent correlation function

$$
G^{C}(\mathbf{r}, t)=\frac{1}{N} \sum_{i, j}\left\langle\delta\left(\mathbf{r}+\mathbf{r}_{i}(0)-\mathbf{r}_{j}(t)\right)\right\rangle_{O},
$$

where $\left\langle\delta\left(\mathbf{r}+\mathbf{r}_{i}(0)-\mathbf{r}_{j}(t)\right)\right\rangle_{C}$ denotes the classical thermal average. (In this way, scattering data can be used to give a physical picture of the scattering system $^{10}$ or alternatively, cross sections can be calculated from a knowledge of the classical mechanics of the scattering system.11)

Following the approach of Aamodt et al., ${ }^{12}$ we consider from Eq. (41b)

$$
\begin{aligned}
\chi_{i j}(\mathbf{q}, t)= & \operatorname{Tr} \rho \exp \left[-i \mathbf{q} \cdot \mathbf{R}_{i}(0) / \hbar\right] \\
\times \exp \left[i \mathbf{q} \cdot \mathbf{R}_{j}(t) / \hbar\right] & \\
= & \int d p^{\prime} d r^{\prime} A_{i}\left(r^{\prime}, p^{\prime}\right) B_{j}\left(r^{\prime}, p^{\prime}, t\right),
\end{aligned}
$$

where, from the application of the Wigner rules derived in the previous section, we have

$$
\begin{aligned}
A_{i}(r, p) & =f_{w}(r, p) \exp (\hbar \Lambda / 2 i) \exp \left(-i \mathbf{q} \cdot \mathbf{r}_{i} / \hbar\right) \\
& =\exp \left(-i \mathbf{q} \cdot \mathbf{r}_{i} / \hbar\right) \exp \left(-\frac{1}{2} \mathbf{q} \cdot \nabla_{\mathbf{p}_{i}^{\prime}}^{\prime}\right) f_{w}(r, p)
\end{aligned}
$$

and

$B_{j}(r, p, t)=\exp \left[(2 t / \hbar) H_{w} \sin (\hbar \Lambda / 2)\right] \exp \left(i \mathbf{q} \cdot \mathbf{r}_{j} / \hbar\right)$,

$$
\begin{aligned}
& =\exp \left\{(t / m) \mathbf{p} \cdot \vec{\nabla}_{r}-(2 t / \hbar) \Phi(r)\right. \\
& \left.\quad \times \sin (\hbar / 2) \overleftarrow{\nabla}_{r} \cdot \vec{\nabla}_{p}\right\} \exp \left(i \mathbf{q} \cdot \mathbf{r}_{j} / \hbar\right) .
\end{aligned}
$$

[In the last line we have used a special form for the Hamiltonian, namely, $H_{w}=p^{2} / 2 m+\Phi(r)$.] Further, we observe

$$
\chi(\mathbf{q}, t)=\frac{1}{N} \sum_{i, j} \chi_{i j}(\mathbf{q}, t) .
$$

Here we consider only the lowest-order contributions in $\hbar,{ }^{13}$ so that $f_{w}$ can be taken proportional to $e^{-\beta H_{v}}$ (cf. Appendix A). We then can write

$$
\begin{aligned}
A_{i}(r, p)=f_{w} & \exp \left(-\beta \mathbf{q}^{2} / 8 m\right) \\
& \times \exp \left(\beta \mathbf{q} \cdot \mathbf{p}_{i} / 2 m\right) \exp \left(-i \mathbf{q} \cdot \mathbf{r}_{\mathbf{i}} / \hbar\right),
\end{aligned}
$$

10 B. N. Brockhouse in Proceedings of the Symposium on Inelastic Scattering of Neutrons in Solids and Liquids (International Atomic Energy Commission, Vienna, 1960).

11 R. Nossal, Phys. Rev. 135, A1579 (1964).

12 R. Aamodt, K. M. Case, M. Rosenbaum, and P. F. Zweifel, Phys. Rev. 126, 1165 (1962).

13 Higher-order corrections are studied in a paper by $M$. Rosenbaum and P. F. Zweifel, Phys. Rev. 137, B271 (1965); Also see M. Rosenbaum, Doctoral Thesis, University of Michigan (1964). 
where we have used the Taylor's series property

$$
\exp (-a \cdot \nabla p) f(p)=f(p-a) .
$$

To the same order

$$
\begin{aligned}
B_{j}(r, p, t) & =\exp \left(t H_{w o} \Lambda\right) \exp \left(i q \cdot \mathbf{r}_{j} / \hbar\right) \\
& =\exp \left[i q \cdot \mathbf{r}_{j}(t) / \hbar\right] .
\end{aligned}
$$

Thus to lowest order

and

$$
\begin{aligned}
\chi(\mathbf{q}, t)= & \frac{1}{N} \sum_{i j} \int d p^{\prime} d r^{\prime} f_{w} \exp \left(-\beta \mathbf{q}^{2} / 8 m\right) \\
& \times \exp \left(\beta \mathbf{q} \cdot \mathbf{p}_{i} / 2 m\right) \exp \left(-i \mathbf{q} \cdot \mathbf{r}_{i} / \hbar\right) \\
& \times \exp \left(i \mathbf{q} \cdot \mathbf{r}_{j}(t) / \hbar\right],
\end{aligned}
$$

$$
\begin{aligned}
G(\mathbf{r}, t)=\frac{1}{N} & (2 \pi \hbar)^{-3} \int d^{3} q \exp (-i \mathbf{q} \cdot \mathbf{r}) \\
& \times \exp \left(-\beta \mathbf{q}^{2} / 8 m\right)\left\langle\exp \left(\beta \mathbf{q} \cdot \mathbf{p}_{i} / 2 m\right)\right. \\
& \left.\times \exp \left(-i \mathbf{q} \cdot \mathbf{r}_{i} / \hbar\right) \exp \left[i \mathbf{q} \cdot \mathbf{r}_{j}(t) / \hbar\right]\right\rangle .
\end{aligned}
$$

Suppose, in Eq. (51), we replace $\mathbf{r}_{i}(0)+i \mathbf{p}_{i}(0) \beta \hbar / 2 m$ by $\mathbf{r}_{i}\left(\frac{1}{2} i \beta \hbar\right)$, which is correct to order $\hbar^{2}$. Then

$$
\begin{aligned}
G(\mathbf{r}, t)= & (2 \pi \hbar)^{-3} N^{-1} \int d^{3} q \exp (-i \mathbf{q} \cdot \mathbf{r}) \\
& \times \exp \left(-\beta \mathbf{q}^{2} / 8 m\right)\left\langle\exp \left[i \mathbf{q} \cdot \mathbf{r}_{j}(t) / \hbar\right]\right. \\
& \left.\times \exp \left[-i \mathbf{q} \cdot \mathbf{r}_{i}\left(\frac{1}{2} i \beta \hbar\right)\right]\right\rangle \\
= & (2 \pi \hbar)^{-3} N^{-1} \int d^{3} q \exp (-i \mathbf{q} \cdot \mathbf{r}) \\
& \times \exp \left(-\beta \mathbf{q}^{2} / 8 m\right)\left\langle\exp \left[-i \mathbf{q} \cdot \mathbf{r}_{i}(0)\right]\right. \\
& \left.\quad \times \exp \left[i \mathbf{q} \cdot \mathbf{r}_{j}\left(t-\frac{1}{2} i \beta \hbar\right)\right]\right\rangle .
\end{aligned}
$$

To obtain Eq. (52b) time-translational invariance has been employed.

Referring now to (39b), we see, after some trivial manipulations, that

$$
S(\mathbf{q}, \epsilon)=\exp (\beta \epsilon / 2) \exp \left(-\beta q^{2} / 8 m\right) S_{C}(\mathbf{q}, \epsilon),
$$

where $S_{C}(\mathbf{q}, \epsilon)$ is related to $G_{C}(\mathbf{r}, t)$ through Eq. (39b). This is the desired result (to lowest order in $\hbar$ ) since it expresses the cross section in terms of the Fourier transform of the classical time-dependent correlation function. It is referred to as the "quasi-classical" approximation.

The integration of Eqs. (52) can be carried out explicitly, to give

$$
\begin{aligned}
G(\mathbf{r}, t) & =\frac{1}{N}\left(\frac{2 m}{\pi \hbar}\right)^{\frac{3}{2}} \\
\times & \sum_{i j}\left\langle\exp \left\{-\left(2 m / \beta \hbar^{2}\right)\left[\mathbf{r}+\mathbf{r}_{i}(0)-\mathbf{r}_{j}\left(t-\frac{1}{2} i \beta \hbar\right)\right]\right\} .\right.
\end{aligned}
$$

Now let us consider the scattering law as $\hbar \rightarrow 0$;

$S(\mathbf{q}, \epsilon)=(2 \pi \hbar)^{-1} \int d t \exp (-i \epsilon t / \hbar)$

$$
\times \sum_{i, j} \int d p d r A_{i} B_{j} .
$$

Changing the dummy variable by $t=\hbar \tau$, and utilizing (44) and (45), we obtain

$$
\begin{aligned}
S(q, \epsilon)= & \exp \left(-\beta q^{2} / 8 m\right) \sum_{i, j} \int d p d r f_{w} \\
& \times \exp \left(\beta \mathbf{q} \cdot \mathbf{p}_{i} / 2 m\right) \exp \left(-i \mathbf{q} \cdot \mathbf{r}_{i} / \hbar\right) \\
& \times(2 \pi)^{-1} \int d \tau \exp (-i \epsilon \tau) \\
& \times \exp \left[i \mathbf{q} \cdot \mathbf{r}_{j}(\tau \hbar) / \hbar\right] .
\end{aligned}
$$

Assuming the limit $\hbar \rightarrow 0$ can be taken before the integrations, we can replace $\mathbf{r}_{j}(\tau \hbar) \rightarrow \mathbf{r}_{j}+\tau \hbar \mathbf{p}_{j} / m$ to get

$$
\begin{aligned}
S(\mathbf{q}, \epsilon)= & \exp \left(-\beta q^{2} / 8 m\right) \exp (\beta \epsilon / 2) \sum_{i, j} \int d p d r f_{w} \\
& \times \exp \left[i \mathbf{q} \cdot\left(\mathbf{r}_{j}-\mathbf{r}_{i}\right) / \hbar\right] \delta\left(\epsilon-\mathbf{q} \cdot \mathbf{p}_{i} / m\right) \\
= & \frac{1}{N} S^{0}(\mathbf{q}, \epsilon) \sum_{i, j} \int_{\times \exp \left[i \mathbf{q} \cdot\left(\mathbf{r}_{j}-\mathbf{r}_{i}\right) / \hbar\right]} d r n_{N}
\end{aligned}
$$

where

$$
\begin{aligned}
& S^{0}(q, \epsilon)=N\left(m \beta / 2 \pi q^{2}\right)^{\frac{1}{2}} \exp \left(-\beta q^{2} / 8 m\right) \\
& \times \exp (\beta \epsilon / 2) \exp \left(-m \beta \epsilon^{2} / 2 q^{2}\right)
\end{aligned}
$$

is the scattering law corresponding to the ideal gas, and $n_{N}=\int d p f_{w}$. It is noted that the "self-terms", i.e., $i=j$, give the ideal gas result. For the case of binary central potential we obtain (with $\rho=N / V$ )

$$
\begin{aligned}
S(\mathbf{q}, \epsilon)= & S^{0}(\mathbf{q}, \epsilon)\left[1+\rho^{-1} \int d^{3} r n_{2}(\mathbf{r}, 0)\right. \\
& \times \exp (i \mathbf{q} \cdot \mathbf{r} / \hbar)] \\
= & S^{0}(\mathbf{q}, \epsilon)\left[1+\rho \int d^{3} r g(r) \exp (i q \cdot \mathbf{r} / \hbar)\right]
\end{aligned}
$$

where $n_{s}\left(\mathbf{r}_{1}, \cdots, \mathbf{r}_{s}\right)$ is the $s$-particle reduced distribution function in configuration space:

$$
n_{s}\left(\mathbf{r}_{1}, \cdots, \mathbf{r}_{s}\right)=[N ! /(N-s) !] \int n_{N} d^{3} r_{s+1} \cdots d^{3} r_{N},
$$

and $g(r)=\rho^{-2} n_{2}(\mathbf{r}, 0)$ is the usual radial distribution function. In deriving (59), we have also used the translational invariance property, viz., for any $\mathbf{a}$,

$$
n_{s}\left(\mathbf{r}, \cdots, \mathbf{r}_{s}\right)=n_{s}\left(\mathbf{r}+\mathbf{a}, \cdots, \mathbf{r}_{s}+\mathbf{a}\right),
$$

which follows from the homogeneity of the scattering system. 
For an ideal gas $n_{N}=V^{-N} ; n_{2}=N(N-1) V^{-2}$; thus $S(q, \epsilon)=S^{0}(q, \epsilon)$ if one drops the contribution of the "distinct terms" $(i \neq j)$ which corresponds to the incident beam $(q=0, \epsilon=0)$ in the first Born approximation. ${ }^{12,13}$

The point here is that if the limit $\hbar \rightarrow 0$ is taken before the $t$ (or $\tau$ ) or $(\mathbf{r}, t)$ integrations are performed, one gets a manifestly incorrect result, i.e., basically the ideal gas result for any system. It is important to go to the limit correctly, i.e., to use the "quasiclassical" approximation.

\section{SOME CONSIDERATIONS FOR THE INCLUSION OF EXCHANGE EFFECTS}

In previous sections, we have assumed the Boltzmann statistics to hold in the system at hand, so that the exchange effects due to the symmetry of the system have been ignored. We now outline a method which enables one to modify the previous results to include such effects.

For simplicity, we assume that the particles in the system are all identical, so that the state vectors (for example, in the coordinate representation) can be written as

$$
|r\rangle^{\theta}=(N !)^{-\frac{1}{2}} \sum_{P} \theta^{|P|}|P r\rangle,
$$

where the summation is over all permutations $P$ of $\mathbf{r}, \cdots, \mathbf{r}_{N},|P|$ is the parity of $P$, and $\theta=1$ for bosons and $\theta=-1$ for fermions.

The symmetrized (or antisymmetrized) Wigner equivalent of an operator $A$ is then written as

$$
\begin{aligned}
A_{w}^{\theta}(r, p)= & \int d z \exp (i p \cdot z / h) \\
& \times\left\langle r-\frac{z}{2}|A| r+\frac{z}{2}\right\rangle^{\theta} / N ! \\
= & \int d k \exp (-i r \cdot k / \hbar) \\
& \times\left\langle p-\frac{k}{2}|A| p+\frac{k}{2}\right\rangle^{\theta} / N !
\end{aligned}
$$

Our aim in this section is to express $A_{w}^{\theta}$ in terms of the previously defined quantity $A_{w}$.

Let us associate with every $P$ an operator $U_{P}$ defined as

$$
U_{P}|r\rangle=|P r\rangle .
$$

It is readily seen that $U_{P}$ is unitary, namely $U_{P}^{\dagger}=$ $U_{P^{-1}}=U_{P}^{-1}$. Also, let

$$
I^{\theta}=(1 / N !) \sum_{P} \theta^{|P|} U_{P}
$$

It can be verified that $I^{\theta}$ is Hermitian, and also that

$$
\begin{gathered}
U_{P} I^{\theta}=\theta^{|P|} I^{\theta}=I^{\theta} U_{P}, \\
I^{\theta} I^{\theta}=I^{\theta}, \\
I^{\theta} A I^{\theta}=A^{S} I^{\theta}=I^{\theta} A^{S},
\end{gathered}
$$

where $A^{S}=(1 / N !) \sum_{P} U_{P} A$. If $A$ is symmetrical (that is, $U_{P} A=A$, for all $P$ ) then $A^{S}=A$ (as is the case for all observable operators). We thus drop the superscript $S$.

In this notation, the matrix elements

$$
\begin{aligned}
{ }^{\theta}\left\langle r|A| r^{\prime}\right\rangle^{\theta} & =N !\left\langle r\left|I^{\theta} A I^{\theta}\right| r^{\prime}\right\rangle \\
& =N !\left\langle r\left|A I^{\theta}\right| r^{\prime}\right\rangle .
\end{aligned}
$$

Therefore, from Eq. (63), we obtain, using (25a),

$$
A_{i p}^{\theta}(r, p)=A_{w}(r, p) \exp (\hbar \Lambda / 2 i) I_{v o}^{\theta}(r, p),
$$

where $I_{w}^{\theta}(r, p)$ is the symmetrized Wigner equivalent of the identity operator [i.e., Eq. (63) for $A=I$ ], or it is the ordinary Wigner equivalent of $I^{\theta}$.

In order to calculate the expectation value of an operator $A$, we consider the definition

$$
\langle A\rangle=\sum_{i} w_{i}\left\langle\phi_{i}|A| \phi_{i}\right\rangle .
$$

Because of the symmetry of the system, the state vectors in the position representation possess the invariance property

for any $P$. Thus

$$
\begin{aligned}
\left\langle r \mid \phi_{i}\right\rangle & =\theta^{|P|}\left\langle P r \mid \phi_{i}\right\rangle \\
& =\theta^{|P|}\left\langle r\left|U_{p}\right| \phi_{i}\right\rangle
\end{aligned}
$$

$$
\begin{array}{r}
\langle A\rangle=(1 / N !)^{2} \sum_{P^{\prime}} \sum_{P} \theta^{|P|+\left|P^{\prime}\right|} \sum_{i} w_{i} \int d r d r^{\prime}\left\langle P r \mid \phi_{i}\right\rangle \\
\times\left\langle\phi_{i} \mid P^{\prime} r^{\prime}\right\rangle\left\langle r^{\prime}|A| r\right\rangle, \\
\langle A\rangle=(1 / N !) \int d r d r^{\prime \theta}\left\langle r^{\prime}|\rho| r\right\rangle^{\theta}\left\langle r|A| r^{\prime}\right\rangle,
\end{array}
$$

where we have used the definition (2). With Eq. (67), we thus have

$$
\langle A\rangle=\operatorname{Tr} \rho I^{\theta} A,
$$

since $\rho$ is symmetrical. Using our previous results, we get

$$
\langle A\rangle=\int d r d p f_{w}^{\theta}(r, p) A_{w}(r, p),
$$

where

$$
\begin{aligned}
f_{w}^{\theta} & =(2 \pi \hbar)^{-3 N} \rho_{w}(r, p) \exp (\hbar \Lambda / 2 i) I_{w}^{\theta}(r, p) \\
& =(2 \pi \hbar)^{-3 N} I_{w}^{\theta}(r, p) \cos \frac{\hbar \Lambda}{2} \rho_{w}(r, p) .
\end{aligned}
$$

In the last step we have used the relation $I^{\theta} \rho=\rho I^{\theta}$. Alternatively we can write

$$
\langle A\rangle=\int d r d p f_{w}(r, p) A_{w}^{\theta}(r, p) .
$$


We thus have reduced the calculation of the exchange effects to the evaluation of the quantity

$$
\begin{aligned}
& N ! I_{w}^{\theta}= \sum_{P} \theta^{|P|} \int d z \exp (i p \cdot z \mid \hbar) \\
& \quad \quad \delta\left(r-P r-\frac{1}{2} z-\frac{1}{2} P z\right) \\
&= \sum_{P} \theta^{|P|} \int d k \exp (-i k \cdot r / \hbar) \\
& \quad \times \delta\left(p-P p-\frac{1}{2} k-\frac{1}{2} P k\right) .
\end{aligned}
$$

Let us note that $I_{v}^{\theta}$ is an even function with respect to both $r$ and $p$. This can be seen by using the property $\delta\left(r-P r^{\prime}\right)=\delta\left(r^{\prime}-P^{-1} r\right)$ and changing the dummy variables $z \rightarrow-z, P \rightarrow P^{-1}$ in (76a). This manipulation shows that the factor $\exp (i p \cdot z / \hbar)$ in the integrand can be replaced by $\cos p \cdot z / \hbar$ (therefore $I_{w}^{\theta}$ is real). Similar manipulations on (76b) yield the symmetry property with respect to $r$.

Secondly, we observe that the term in Eqs. (76) corresponding to the identity permutation is 1 , which corresponds in turn to Boltzmann statistics. [The factor $N !$ is to be replaced by $(1)^{N}=1$ in the latter statistics, since all particles are distinct.] The evaluation of $I_{w}^{\theta}$ as a power series in $\hbar$ is not appropriate because of the essential singularity at $\hbar=0$. One can reduce this evaluation to the calculation of the contributions of cyclic permutations.

\section{Second Quantized Approach ${ }^{14}$}

An alternative way to handle the problem of inclusion of the exchange effects is to utilize the second quantized formalism. The (anti)symmetrized position ket vectors can be written as

$$
I^{\theta}|r\rangle=(N !)^{-\frac{1}{2}} \psi^{\dagger}\left(\mathbf{r}_{1}\right) \cdots \psi^{\dagger}\left(\mathbf{r}_{N}\right)|0\rangle
$$

where $\psi(r)$ and its Hermitian conjugate $\psi^{\dagger}(\mathbf{r})$ are the annihilation and creation operators which satisfy the usual (anti)commutation relations corresponding to (fermions) bosons. The matrix elements

$$
\begin{aligned}
& { }^{\theta}\left\langle r^{\prime}|A| r\right\rangle^{\theta} \\
& =\left\langle 0\left|\psi\left(\mathbf{r}_{N \rightarrow 1}^{\prime}\right) \cdots \psi\left(\mathbf{r}_{N \rightarrow 1}^{\prime}\right) A \psi^{\dagger}\left(\mathbf{r}_{1}\right) \cdots \psi^{\dagger}\left(\mathbf{r}_{N}\right)\right| 0\right\rangle \\
& =\operatorname{Tr} A \psi^{\dagger}\left(\mathbf{r}_{1}\right) \cdots \psi\left(\mathbf{r}_{N}\right) \psi\left(\mathbf{r}_{N}^{\prime}\right) \cdots \psi\left(\mathbf{r}_{1}^{\prime}\right),
\end{aligned}
$$

where we have used the fact that the total number of particles, $N$, in the system is constant. Substituting in Eq. (73), and utilizing (20g) we obtain

where

$$
\langle A\rangle=\int d r d p f_{w}^{\theta}(r, p, t) A_{w}(r, p),
$$

$$
\begin{aligned}
f_{w}^{\theta}(r, p, t)= & (2 \pi \hbar)^{-3 N}(N !)^{-1} \int d z \exp (i p \cdot z / \hbar) \\
& \times \operatorname{Tr} \rho(t) \psi^{\dagger}\left(\mathbf{r}_{1}+\frac{1}{2} \mathbf{z}_{1}\right) \cdots \psi^{\dagger}\left(\mathbf{r}_{N}+\frac{1}{2} \mathbf{z}_{N}\right) \\
& \times \psi\left(\mathbf{r}_{N}-\frac{1}{2} \mathbf{z}_{N}\right) \cdots \psi\left(\mathbf{r}_{1}-\frac{1}{2} \mathbf{z}_{1}\right), \quad(80)
\end{aligned}
$$

14 For a general review, see W. E. Brittin and W. R. Chappell, Rev. Mod. Phys. 34, 620 (1962); also R. Balescu, Statistical Mechanics of Charged Particles (Interscience Publishers, Inc., New York, 1963), Part II. which represents the Wigner distribution function in the second quantized formalism.

Equation (80) can also be written in the Heisenberg picture

$$
\begin{aligned}
f_{w}^{\theta}(r, p, t)= & (N !)^{-1}(2 \pi \hbar)^{-3 N} \int d z \exp (i p \cdot z / \hbar) \\
& \times \operatorname{Tr} \rho(0) \Gamma^{\dagger}\left(r+\frac{1}{2} z, t\right) \Gamma\left(r-\frac{1}{2} z, t\right),
\end{aligned}
$$

where

$$
\Gamma(r, t)=\psi\left(\mathbf{r}_{N}, t\right) \cdots \psi\left(\mathbf{r}_{1}, t\right) .
$$

Let us assume, for simplicity, that

$$
\begin{gathered}
H_{w}=p^{2} / 2 m+\Phi(r), \\
\Phi(r)=\sum_{i<j} \phi\left(r_{i}-r_{j}\right) .
\end{gathered}
$$

(We also assume that the interaction potential is real.) One then has

$i \hbar \partial \psi(\mathbf{r}, t) / \partial t=-\left(\hbar^{2} / 2 m\right) \nabla_{r}^{2} \psi(\mathbf{r}, t)+\hat{\phi}(\mathbf{r}) \psi(\mathbf{r}, t)$,

where

$$
\hat{\phi}(\mathbf{r})=\int d^{8} r^{\prime} \phi\left(\mathbf{r}-\mathbf{r}^{\prime}\right) \psi^{\dagger}\left(\mathbf{r}^{\prime}\right) \psi\left(\mathbf{r}^{\prime}\right)
$$

Thus, $\Gamma(r, t)$ obeys the equation (with $\nabla_{r}^{2}=\sum_{i} \nabla_{\mathbf{r}_{i}}^{2}$ ), $i \hbar \partial \Gamma(r, t) / \partial t=-\left(\hbar^{2} / 2 m\right) \nabla_{r}^{2} \Gamma(r, t)+\Phi(r) \Gamma(r, t)$

$$
+\sum_{j=1}^{N} \hat{\phi}\left(\mathbf{r}_{j}\right) \Gamma(r, t) \theta^{N-j},
$$

where we have successively used the (anti)commutator relation

$$
\left[\psi(\mathbf{r}), \hat{\phi}\left(\mathbf{r}^{\prime}\right)\right]_{\theta}=\psi(\mathbf{r}) \phi\left(\mathbf{r}^{\prime}-\mathbf{r}\right) .
$$

The last term in the right-hand side of Eq. (86) vanishes identically since there is no $(N+1)$-particle state. One readily finds

$$
\begin{aligned}
& i \hbar \frac{\partial}{\partial t} \Gamma^{\dagger}\left(r+\frac{1}{2} z, t\right) \Gamma\left(r-\frac{1}{2} z, t\right) \\
& =\left[\hbar^{2} / 2 m \nabla_{z} \cdot \nabla_{r}-2 \Phi(r) \sinh \frac{1}{2} \overleftarrow{\nabla}_{r} \cdot z\right] \\
& \times \Gamma^{\dagger}\left(r+\frac{z}{2}, t\right) \Gamma\left(r-\frac{z}{2}, t\right) \text {. }
\end{aligned}
$$

Equation (88) enables one to calculate the time rate of change of $f_{w}^{\theta}$ to obtain

$$
\begin{aligned}
\left(\frac{\partial}{\partial t}+\frac{1}{m} p \cdot \nabla_{r}-\frac{2}{\hbar} \phi(r) \sin \frac{\hbar}{2} \overleftarrow{\nabla}_{r} \cdot \vec{\nabla}_{p}\right) \\
\\
\times f_{w}^{\theta}(r, p, t)=0,
\end{aligned}
$$

which is nothing but the quantum Liouville equation [cf. Eq. (30)].

In kinetic theory, one introduces the reduced distribution functions by integrating $f_{w}^{\theta}$ with respect to all state variables $\mathbf{r}, \mathbf{p}$ but those belonging to one, 
two, etc., particles. Let us define with $i \triangleq\left(\mathbf{r}_{i}, \mathbf{p}_{i}\right)$,

$$
\begin{aligned}
f_{s}^{\theta}(1, \cdots, s ; t) & =\frac{N !}{(N-s) !} \\
& \times \int d^{3} r_{s+1} \cdots d^{3} r_{N} d_{3} p_{s+1} \cdots d^{3} p_{N} f_{w}^{\theta},
\end{aligned}
$$

where we have used the "generic" normalization. By integrating Eq. (89) one obtains the quantum BBGKY hierarchy:

$$
\begin{gathered}
\left(\frac{\partial}{\partial t}+\frac{1}{m} p \cdot \nabla_{r}-\frac{2}{\hbar} \sum_{i<j \leq s} \phi\left(\mathbf{r}_{i}-\mathbf{r}_{j}\right) \sin \frac{1}{2} \hbar \bar{\nabla}_{r} \cdot \vec{\nabla}_{p}\right) \\
\times f_{s}^{\theta}(1, \cdots, s ; t)=\frac{2}{\hbar} \sum_{i=1}^{s} \int d^{3} r_{s+1} d^{3} p_{s+1} \phi\left(\mathbf{r}_{i}-\mathbf{r}_{s+1}\right) \\
\quad \times \sin \left(\frac{1}{2} \hbar \bar{\nabla}_{r} \cdot \vec{\nabla}_{p}\right) f_{s+1}^{\theta}(1, \cdots, s+1 ; t) .
\end{gathered}
$$

The right-hand side of Eq. (90) stems from the contribution of the last term in Eq. (86) which does not vanish when $s<N$.

Equation (90) [rather the first one (or two) of the chain] has been studied for various systems in which the radiation field, photon field, spin and relativistic effects are included. ${ }^{15}$ For further details, the reader is referred to Ref. 15 .

\section{ACKNOWLEDGMENTS}

Two of the authors (M. R. and P. F. Z.) gratefully acknowledge many stimulating discussions with Professor R. K. Osborn and Professor F. C. Shure during the early stages of this work. In addition, P. F. Z. wishes to express his gratitude to the Ford Foundation for financial support and to the Çekmece Nuclear Center for its hospitality during the time this paper was being written.

\section{APPENDIX A}

\section{Solution of Bloch Equation}

Although Eqs. (37) can be formally solved to obtain

$$
\Omega_{w}=\exp \left(-\beta H_{w} \cos \hbar \Lambda / 2\right),
$$

the evaluation of this result is somewhat lengthy. Instead, here, for simplicity, we restrict ourselves to the case for which

$$
H_{w}=p^{2} / 2 m+\Phi(r),
$$

where $\Phi(r)$ is the interaction potential which is usually assumed to be pairwise additive; i.e.,

$$
\Phi(r)=\sum_{i<j} \phi\left(\left|\mathbf{r}_{i}-\mathbf{r}_{j}\right|\right) \text {. }
$$

\footnotetext{
15 R. K. Osborn and E. H. Klevans, Ann. Phys. (N.Y.) 15, 105 (1961); E. Ozizmir, Doctoral thesis, University of Michigan (1962); R. K. Osborn, Phys. Rev. 130, 2142 (1963).
}

The Bloch equation for this case can be written as

$$
\begin{aligned}
\partial \chi / \partial \beta=\left(\hbar^{2} / 8 m\right) e^{\beta \phi} \nabla_{r}^{2} e^{-\beta \phi} \chi+\phi e^{\beta \nu^{2} / 2 m} \\
\times\left(1-\cos \frac{1}{2} \hbar \bar{\nabla}_{r} \cdot \vec{\nabla}_{p}\right) e^{-\beta \nu^{2} / 2 m},
\end{aligned}
$$

where we have set

$$
\chi=e^{\beta H^{*}} \Omega_{w} .
$$

The first term in the right-hand side of (4) can be written as

$$
\left(\hbar^{2} / 8 m\right)\left[\nabla_{r}^{2}-2 \beta \nabla_{r} \phi \cdot \nabla_{r}-\beta \nabla_{r}^{2} \phi+\beta^{2}\left(\nabla_{r} \phi\right)^{2}\right] x .
$$

We also have

$$
\begin{gathered}
\exp \left(\beta p^{2} / 2 m\right) \cos \left(\frac{1}{2} \hbar\right) \widehat{\nabla}_{r} \cdot \vec{\nabla}_{\nu} \exp \left(-\beta p^{2} / 2 m\right) \\
=\exp \left(\beta p^{2} / 2 m\right) \operatorname{Re} \exp \left(\frac{1}{2} i \hbar \widehat{\nabla}_{r} \cdot \vec{\nabla}_{p}\right) \exp \left(-\beta p^{2} / 2 m\right) \\
=\exp \left(\beta p^{2} / 2 m\right) \operatorname{Re} \exp \left[-\beta\left(p+\frac{1}{2} i \hbar \widehat{\nabla}_{r}\right) / 2 m\right] \\
\times \exp \left(\frac{1}{2} i \hbar \hat{\nabla}_{r} \cdot \vec{\nabla}_{p}\right) \\
=\exp \left(\beta \hbar^{2} \overleftarrow{\nabla}_{r}^{2} / 8 m\right) \operatorname{Re} \exp \left(-i \beta \hbar \widehat{\nabla}_{r} \cdot p / 2 m\right) \\
\times \exp \left(i \hbar \hat{\nabla}_{r} \cdot \vec{\nabla}_{p} / 2\right) .
\end{gathered}
$$

Hence, one obtains, with $\chi(\beta=0)=1$,

$$
\begin{aligned}
\partial \chi / \partial \beta= & \left(\hbar^{2} / 8 m\right)\left[\nabla_{r}^{2} \chi-2 \beta \nabla_{r} \phi \cdot \nabla_{r} \chi\right. \\
& \left.-\beta\left(\nabla_{r}^{2} \phi\right) \chi+\beta^{2}\left(\nabla_{r} \phi\right)^{2} \chi\right]+\phi \chi \\
& -\left[\exp \left(\hbar^{2} \beta \nabla_{r}^{2} / 8 m\right) \phi\right]\left[\cos (\hbar \beta / 2 m) \hat{\nabla}_{r}\right. \\
& \cdot p \cos \left(\frac{1}{2} \hbar\right) \hat{\nabla}_{r} \cdot \vec{\nabla}_{p}+\sin (\hbar \beta / 2 m) \hat{\nabla}_{r} \\
& \left.\cdot p \sin \left(\frac{1}{2} \hbar\right) \hat{\nabla}_{r} \cdot \vec{\nabla}_{p}\right] \chi
\end{aligned}
$$

This equation can be solved by expanding $\chi$ in powers of $\hbar^{2}$. To the lowest order, $\chi_{0}=1$, which corresponds to the classical case. To first order, one gets

$$
\chi_{1}=(8 m)^{-1}\left[-\beta^{2} \nabla^{2} \phi+\frac{1}{3} \beta^{3}(p \cdot \nabla)^{2} \phi\right],
$$

which was first derived by Wigner. ${ }^{2}$ The second-order contribution can also be carried out although the calculation is somewhat lengthy. The result is

$$
\begin{aligned}
\chi_{2}= & \left(\beta^{3} / 64 m^{2}\right)\left\{-\frac{1}{2} \nabla^{4} \phi+\beta\left[\frac{1}{6} \nabla^{2}(\nabla \phi)^{2}\right.\right. \\
& \left.+(1 / 3 m)(p \cdot \nabla)^{2} \nabla^{2} \phi+\frac{1}{3} \nabla \phi \cdot \nabla\left(\nabla^{2} \phi\right)+\frac{1}{2}\left(\nabla^{2} \phi\right)^{2}\right] \\
& -\beta^{2}\left[\frac{2}{15} \nabla \phi \cdot \nabla(\nabla \phi)^{2}+(2 / 15 m) \nabla \phi \cdot \nabla(p \cdot \nabla)^{2} \phi\right. \\
& +\frac{1}{3}\left(\nabla^{2} \phi\right)(\nabla \phi)^{2}+(1 / 3 m)\left(\nabla^{2} \phi\right)(p \cdot \nabla)^{2} \phi \\
& \left.+(4 / 15 m)(\nabla(p \cdot \nabla \phi))^{2}+\frac{1}{30}(p \cdot \nabla)^{4} \phi\right] \\
& +\beta^{3}\left[\frac{1}{18}(\nabla \phi)^{4}+(1 / 9 m)(\nabla \phi)^{2}(p \cdot \nabla)^{2} \phi\right. \\
& \left.\left.+\left(1 / 6 m^{2}\right)\left((p \cdot \nabla)^{2} \phi\right)^{2}\right]\right\} .
\end{aligned}
$$

In order to calculate the partition function $Z$ we consider the relation

$$
\int d p d r \Omega_{w}=Z(2 \pi \hbar)^{3 N}
$$

or

$$
Z=(2 \pi \hbar)^{-3 N}\langle\chi\rangle_{C}
$$


one obtains

$$
Z=Z_{C}\left[1+\hbar^{2} C_{1}+\hbar^{4} C_{2}+\cdots\right],
$$

where $Z_{C}$ is the classical partition function. One has

$$
\begin{aligned}
C_{1}= & (1 / 8 m)\left[-\beta^{2}\left\langle\nabla^{2} \phi\right\rangle_{C}+\frac{1}{3} \beta^{3}\left\langle(\nabla \phi)^{2}\right\rangle_{C}\right. \\
& \left.+\frac{1}{3} \beta^{2}\left\langle\nabla^{2} \phi\right\rangle_{C}\right] \\
=-\left(\beta^{2} / 24 m\right)\left\langle\nabla^{2} \phi\right\rangle_{C} . &
\end{aligned}
$$

and

$$
\begin{aligned}
C_{2}= & \left(\beta^{3} / 4 m^{2}\right)\left[\frac{\beta}{288}\left\langle(\nabla \phi)^{4}\right\rangle_{C}-\frac{\beta^{2}}{72}\left\langle\left(\nabla^{2} \phi\right)(\nabla \phi)^{2}\right\rangle_{C}\right. \\
& -\frac{\beta^{2}}{120}\left\langle\nabla \phi \cdot \nabla(\nabla \phi)^{2}\right\rangle_{C}+\frac{\beta}{72}\left\langle\left(\nabla^{2} \phi\right)^{2}\right\rangle_{C} \\
& +\frac{\beta}{45}\left\langle\nabla \phi \cdot \nabla\left(\nabla^{2} \phi\right)\right\rangle_{C} \\
& \left.+\frac{\beta}{180}\left\langle\nabla^{2}(\nabla \phi)^{2}\right\rangle_{C}-\frac{1}{60}\left\langle\nabla^{4} \phi\right\rangle_{C}\right], \\
= & \frac{\beta^{3}}{144 m^{2}}\left[\frac{\beta}{8}\left\langle\left(\nabla^{2} \phi\right)^{2}\right\rangle_{C}+\frac{\beta}{40}\left\langle\nabla^{2}(\nabla \phi)^{2}\right\rangle_{C}\right. \\
& \left.-\frac{7}{40}\left\langle\nabla^{4} \phi\right\rangle_{C}\right] .
\end{aligned}
$$

The evaluation of $C_{2}$ has been carried out by Goldberger and Adams ${ }^{16}$ using field theoretic methods. Our result, however, differs by a factor of $\frac{7}{8}$ from theirs in the middle term of the last equation.

The unsatisfactory part of the above analysis is that these coefficients blow up the limit $N \rightarrow \infty$. This can be seen by writing

$$
\left\langle\nabla^{2} \phi\right\rangle_{C}=N \rho^{-1} \int d^{3} r n_{2}^{C}(r, 0) \nabla^{2} \phi(r)
$$

where $n_{2}^{C}\left(\mathbf{r}_{1}, \mathbf{r}_{2}\right)=n_{2}^{C}\left(\mathbf{r}_{1}-\mathbf{r}_{2}, 0\right)$ is the classical reduced distribution function in configuration space defined as in Eq. (60), and $\rho=N / V$ (= finite). This difficulty was first observed by Mayer and Band. ${ }^{17}$

In order to remedy this difficulty, one can use the well-known property that the free energy per particle $F / N$ is a finite quantity in this limit, as was shown by Kahn and Uhlenbeck ${ }^{18}$ in general terms. In other words, $Z^{1 / N}$ remains finite as $N \rightarrow \infty$ [cf. (A15)]. This can be observed to be true for the case of the harmonic oscillator; cf. Eq. (B10). Green ${ }^{19}$ demonstrated that if one expands $F / N$, instead of $Z$, to which it is related through the relation

$$
Z=e^{-\beta F},
$$

${ }^{16}$ M. L. Goldberger and E. N. Adams, II, J. Chem. Phys. 20, 240 (1952).

17 J. E. Mayer and W. Band, J. Chem. Phys. 15, 141 (1947).

18 B. Kahn and G. E. Uhlenbeck, Physica 5, 399 (1938).

19 H. S. Green, J. Chem. Phys. 19, 955 (1951). then the contribution to order $\hbar^{2}$ remains finite as $N \rightarrow \infty$. We now show that this is also the case for the term of order $\hbar^{4}$.

To do this, we consider

$$
\begin{aligned}
\left(Z / Z_{C}\right)^{1 / N}= & \left(1+\hbar^{2} C_{1}+\hbar^{4} C_{2}+\cdots\right)^{1 / N} \\
= & 1+\hbar^{2}(1 / N) C_{1}+\hbar^{4}\left[(1 / N) C_{2}\right. \\
& \left.+(1 / 2 N)(1 / N-1) C_{1}^{2}\right]+O\left(\hbar^{6}\right) .
\end{aligned}
$$

We have already seen that

$$
(1 / N) C_{1}=-\left(\beta^{2} / 24 m \rho\right) \int d^{3} r n_{2}^{C}(\mathbf{r}, 0) \nabla^{2} \phi(r),
$$

which is bounded as $N \rightarrow \infty$, where $r=|\mathbf{r}|$ (not to be confused with $3 N$-dimensional vector $r$ ).

We can write from (A13) (by making use of a vector identity and an integration by parts)

$$
\begin{aligned}
& (1 / N) C_{2}=\left(\beta^{3} / 1152 m^{2} N\right) \\
& \times\left[\beta\left\langle\left(\nabla^{2} \phi\right)^{2}\right\rangle_{C}+\frac{2 \beta}{5}\left\langle\phi(\widehat{\nabla} \cdot \vec{\nabla})^{2} \phi\right\rangle_{C}-\left\langle\nabla^{4} \phi\right\rangle_{C}\right] .
\end{aligned}
$$

It is readily seen that

$$
\frac{1}{N}\left\langle\nabla^{4} \phi\right\rangle_{C}=\frac{2}{\rho} \int d^{3} r n_{2}^{C}(r, 0) \nabla^{4} \phi(r)
$$

and

$$
\begin{gathered}
\frac{1}{N}\langle\phi(\nabla \cdot \nabla)\rangle_{C}=\frac{2}{\rho} \int d^{3} r n_{2}^{C}(r, 0)\left[\phi(r)\left(\widehat{\nabla}_{\mathbf{r}} \cdot \vec{\nabla}_{\mathbf{r}}\right)^{2} \phi(r)\right] \\
+\frac{1}{\rho} \int d^{3} r d^{3} r^{\prime} n_{3}^{C}\left(\mathbf{r}, \mathbf{r}^{\prime}, 0\right)\left[\phi(r)\left(\hat{\nabla}_{\mathbf{r}} \cdot \vec{\nabla}_{\mathbf{r}^{\prime}}\right)^{2} \phi\left(r^{\prime}\right)\right] .
\end{gathered}
$$

Therefore these quantities are both finite. Finally, we calculate

$$
\begin{aligned}
& \frac{1}{N}\left\langle\left(\nabla^{2} \phi\right)^{2}\right\rangle_{C}=\frac{2}{\rho} \int d^{3} r n_{2}^{C}(r, 0)\left(\nabla^{2} \phi(r)\right)^{2} \\
& \quad+\frac{4}{\rho} \int d^{3} r d^{3} r^{\prime} n_{3}^{C}\left(\mathbf{r}, \mathbf{r}^{\prime}, 0\right) \nabla^{2} \phi(r) \nabla^{2} \phi\left(r^{\prime}\right) \\
& \quad+\frac{1}{\rho} \int d^{3} r d^{3} r^{\prime} d^{3} r^{\prime \prime} n_{4}^{C}\left(\mathbf{r}, \mathbf{r}^{\prime}+\mathbf{r}^{\prime \prime}, \mathbf{r}^{\prime \prime \prime}, 0\right) \nabla^{2} \phi(r) \nabla^{2} \phi\left(r^{\prime}\right)
\end{aligned}
$$

The last term in this equation contains a part which is proportional to $N$. This part, in fact, compensates the term $-(1 / 2 N) C_{1}^{2}$ which also blows up as $N \rightarrow \infty$.

To see this, we introduce the cluster development ${ }^{20}$

\footnotetext{
${ }^{30}$ T. R. Hill, Statistical Mechanics (McGraw-Hill Book Company, Inc., New York, 1956).
} 
as (by dropping the superscript $C$ )

$$
\begin{aligned}
n_{1} & =\rho=G_{1}, \\
n_{2}\left(\mathbf{r}_{1}, \mathbf{r}_{2}\right) & =G_{2}\left(\mathbf{r}_{1}, \mathbf{r}_{2}\right)+\rho^{2} \\
n_{3}\left(\mathbf{r}_{1}, \mathbf{r}_{2}, \mathbf{r}_{3}\right) & =G_{3}\left(\mathbf{r}_{1}, \mathbf{r}_{2}, \mathbf{r}_{3}\right)+\rho\left[G_{2}\left(\mathbf{r}_{1}, \mathbf{r}_{2}\right)\right. \\
& \quad+\text { cyclic terms }]+\rho^{3},
\end{aligned}
$$

etc., where $G_{s}$ is the $s$-particle correlation function which vanishes as $|\mathbf{r}| \rightarrow \infty$. The last term in the righthand side of (A21) becomes, after some manipulations,

$$
\begin{gathered}
=\frac{1}{\rho} \int d^{3} r d^{3} r^{\prime} d^{3} r^{\prime \prime} G_{4}\left(\mathbf{r}, \mathbf{r}^{\prime}+\mathbf{r}^{\prime \prime}, \mathbf{r}^{\prime \prime}, 0\right) \nabla^{2} \phi(r) \nabla^{2} \phi\left(r^{\prime}\right) \\
+\frac{2}{\rho} \int d^{3} r d^{3} r^{\prime} d^{3} r^{\prime \prime} G_{2}\left(\mathbf{r}-\mathbf{r}^{\prime}-\mathbf{r}^{\prime \prime}, 0\right) \\
\times G_{2}\left(\mathbf{r}^{\prime \prime}, 0\right) \nabla^{2} \phi(r) \nabla^{2} \phi\left(r^{\prime}\right) \\
+\frac{V}{\rho}\left[\int d^{3} r n_{2}^{C}(\mathbf{r}, 0) \nabla^{2} \phi(r)\right]^{2} .
\end{gathered}
$$

The last term is the contribution of the part of the double-pair correlations which divides $n_{4}\left(\mathbf{r}, \mathbf{r}^{\prime}+\mathbf{r}^{\prime \prime}\right.$, $\left.\mathbf{r}^{\prime}+\mathbf{r}^{\prime \prime}, \mathbf{r}^{\prime \prime}, 0\right)$ as

$$
G_{2}(\mathbf{r}, 0) G_{2}\left(\mathbf{r}^{\prime}+\mathbf{r}^{\prime \prime}, \mathbf{r}^{\prime \prime}\right)=G_{2}(r, 0) G_{2}\left(r^{\prime}, 0\right) .
$$

This term blows up linearly with $N$. When (A23) is substituted in (A16) one finds that this term cancels the term $C_{1}^{2} / 2 N$; therefore the coefficient of $\hbar^{4}$ remains finite.

The free energy, $F$, can then be written as

$$
\begin{aligned}
F= & F_{C}-(N / \beta) \ln \left(Z / Z_{C}\right)^{1 / N} \\
=F_{C} & -\left(\hbar^{2} / \beta\right) C_{1}-\left(\hbar^{4} / \beta\right) \\
& \times\left[\left(C_{2}-\frac{1}{2} C_{1}^{2}\right)-(1 / N) C_{1}^{2}\right]+O\left(\hbar^{6}\right),
\end{aligned}
$$

a result which may be used to calculate the quantum corrections to the equation of state.

To do this, we consider the definition of pressure ${ }^{20}$

$$
\begin{aligned}
p=-\partial F / \partial V \\
=p^{c}-\frac{\hbar^{2}}{\beta} \frac{\partial C_{1}^{2}}{\partial V}-\frac{\hbar^{4}}{\beta} \frac{\partial}{\partial V} \\
\times\left[\left(C_{2}-\frac{1}{2} C_{1}^{2}\right)-\frac{1}{N} C_{1}^{2}\right]+O\left(\hbar^{6}\right),
\end{aligned}
$$

where $p^{C}$ is the classical pressure which is to be calculated from the classical equation of state.

To evaluate the volume differentiations, one can consider the spatial integrations to be carried out in a box of length $V^{\frac{1}{3}}$. By changing the dummy variable $\mathbf{r}=\mathbf{x} V^{\mathbf{k}}$, so that the volume of integration becomes unity, the differentiation can be performed on the integrand. ${ }^{20}$ In general, one has, for an integrable function $h\left(\mathrm{r}_{1}, \cdots, \mathrm{r}_{8}, V\right)$,

$$
\begin{aligned}
& V \frac{\partial}{\partial V} \int d^{3} r_{1} \cdots d^{3} r_{s} h\left(\mathbf{r}_{1}, \cdots, \mathbf{r}_{s}, V\right) \\
& =(s-1) \int d^{3} r_{1} \cdots d^{3} r_{s} h\left(\mathbf{r}_{1}, \cdots, \mathbf{r}_{s}, V\right) \\
& \quad+\int d^{3} r_{1} \cdots d^{3} r_{s} V \frac{\partial}{\partial V} h\left(\mathbf{r}_{1}, \cdots, \mathbf{r}_{s}, V\right) \\
& \quad+\frac{1}{3} \int d^{3} r_{1} \cdots d^{3} r_{s} \sum_{i=1}^{s} \nabla_{\mathbf{r}_{i}} \cdot\left(\mathbf{r}_{i} \cdot h\left(\mathbf{r}_{1}, \cdots, \mathbf{r}_{s}, V\right)\right)
\end{aligned}
$$

The last term vanishes if $h$ vanishes sufficiently rapidly for large $\left|\mathbf{r}_{\mathbf{1}}\right|$.

Here for simplicity, we consider dilute systems; that is we ignore correlations involving more than two particles. We thus obtain

where

$$
p=p^{C}+\hbar^{2} A_{1}+\hbar^{4} A_{2}+O\left(\hbar^{0}\right),
$$

$$
\begin{aligned}
A_{1}= & \left(\beta \rho^{2} / 24 m\right) \int d^{3} r g(r) \nabla^{2} \phi(r) \\
A_{2}= & -\left(\beta^{2} \rho^{2} / 1152 m^{2}\right)\left\{2 \beta \int d^{3} r g(r)\left(\nabla^{2} \phi\right)^{2}\right. \\
& +4 \beta \rho \int d^{3} r d^{3} r^{\prime} g\left(\left|\mathbf{r}-\mathbf{r}^{\prime}\right|\right) \nabla^{2} \phi(r) \nabla^{2} \phi\left(r^{\prime}\right) \\
& +6 \beta \rho^{2} \int d^{3} r d^{3} r^{\prime} d^{3} r^{\prime \prime} g\left(\left|\mathbf{r}-\mathbf{r}^{\prime}-\mathbf{r}^{\prime \prime}\right|\right) \\
& \times g\left(r^{\prime \prime}\right) \nabla^{2} \phi(r) \nabla^{2} \phi\left(r^{\prime}\right)+\frac{4}{5} \beta \int d^{3} r g(r)\left[\phi(\overleftarrow{\nabla} \cdot \vec{\nabla})^{2} \phi\right] \\
& +\frac{2}{5} \beta \rho \int d^{3} r d^{3} r^{\prime} g\left(\left|\mathbf{r}-\mathbf{r}^{\prime}\right|\right)\left[\phi(r)\left(\overleftarrow{\nabla}_{\mathbf{r}} \cdot \vec{\nabla}_{\mathbf{r}^{\prime}}\right)^{2} \phi\left(r^{\prime}\right)\right] \\
& \left.-\frac{4}{5} \beta \int d^{3} r g(r) \nabla^{4} \phi-4 \rho\left[\int d^{3} r g(r) \nabla^{2} \phi\right]^{2}\right\} . \quad(\mathrm{A} 27)
\end{aligned}
$$

Here $g(r)$ is the radial distribution function defined as $\rho^{2} g(r)=n_{2}(r, 0)$. (We have also dropped the contribution of the volume differentiation of correlations, which may be small for dilute systems.)

Our result, to the order $\hbar^{2}$, coincides with those given by Uhlenbeck and Beth ${ }^{21}$ and also by Green ${ }^{19}$ if one further assumes that

$$
g(r)=\exp [-\beta \phi(r)],
$$

a relation which is valid in the low-density limit. ${ }^{20}$ In the latter case, it is possible to simplify further the quantities in (A27).

${ }^{21}$ G. E. Uhlenbeck and E. Beth, Physica 3, 729 (1936); 4, 915 (1937). For an excellent review of this subject, see J. de Boer, Rept. Progr. Phys. 12, 305 (1949). 


\section{APPENDIX B}

Harmonic Oscillator

For the harmonic oscillator, which is characterized by the Hamiltonian

$$
H_{v}=p^{2} / 2 m+\frac{1}{2} m \omega^{2} r^{2},
$$

the Bloch equation (37) reduces to

$$
\begin{aligned}
\partial \Omega_{w} / \partial \beta=-H_{w} \Omega_{w}+ & \left(\hbar^{2} / 8 m\right) \\
& \times\left[\nabla_{r}^{2}+m^{2} \omega^{2} \nabla_{p}^{2}\right] \Omega_{w} .
\end{aligned}
$$

We seek a solution of (B2) of the form

$$
\Omega_{w}=\exp \left[-A(\beta) H_{w}+B(\beta)\right],
$$

which yields the following equation:

$-(d A / d \beta) H_{w}+(d B / d \beta)$

$$
=-H_{w}-\left(\frac{1}{4} \hbar^{2}\right) \omega^{2} A+\left(\frac{1}{4} \hbar^{2}\right) \omega^{2} H_{w} \text {. }
$$

Since the only $(r, p)$ dependence in (B4) occurs in $H_{w}$, its coefficient must vanish separately. Thus, we obtain

$$
\begin{aligned}
& A=(2 / \hbar \omega) \tanh \hbar \omega \beta / 2, \\
& B=-\ln \cosh \hbar \omega \beta / 2,
\end{aligned}
$$

where the initial condition $\Omega(\beta=0)=1$ has been used.

The partition function can now be calculated by using Eq. (A10), which yields

$$
Z=(1 / \hbar \omega A)^{3 N}[1 / \cosh (\hbar \omega \beta / 2)],
$$

and, therefore

$$
\begin{aligned}
f_{w} & =(1 / 2 \pi \hbar)^{3 N}\left(\frac{1}{2} \Omega_{w}\right) \\
& =(\omega A / 2 \pi)^{3 N} e^{-A(\beta) H_{w}} .
\end{aligned}
$$

Let us note that the free energy per particle is bounded as $N \rightarrow \infty$, since

$$
\begin{aligned}
(F / N) & =-(1 / \beta) \ln Z^{(1 / N)} \\
& =(1 / \beta) \ln (\hbar \omega A)\left[\cosh \left(\frac{1}{2} \hbar \omega \beta\right)\right]^{1 / N}
\end{aligned}
$$

as was pointed out in Appendix A. One obtains

$$
\lim _{N \rightarrow \infty}(F / N)=(1 / \beta) \ln \left[2 \tanh \left(\frac{1}{2} \hbar \omega \beta\right)\right] . \quad(B 10)
$$

